\title{
Studies of the gas volume current density in the ATLAS Resistive Plate Chamber detector during the 2018 data-taking at the LHC
}

\author{
D. Krasnopevtsev ${ }^{a, 1}$ on behalf of ATLAS MUON Collaboration \\ ${ }^{a}$ Department of Modern Physics and State Key Laboratory of Particle Detection and Electronics, University \\ of Science and Technology of China, \\ 96 Jinzhai Road, Hefei 230026, Anhui China \\ E-mail: Dimitriy.Krasnopevtsev@cern.ch
}

\begin{abstract}
The ATLAS Resistive Plate Chamber (RPC) detector is a tracking detector, used to primarily trigger muons in the ATLAS barrel region (which corresponds to a pseudorapidity range of $|\eta|<1.05$ ) at the $40 \mathrm{MHz}$ collision rate, and to provide $\eta$ and $\phi$ coordinates. The RPC system consists of about 3700 gas volumes covering a sensitive surface of about $4000 \mathrm{~m}^{2}$. It is arranged in three concentric doublet layers at radius $7 \mathrm{~m}, 8 \mathrm{~m}$ and $10 \mathrm{~m}$, and operating in an approximately 0.5-1 Tesla toroidal magnetic field. RPCs provide up to 6 space points along the muon track with a space-time resolution of about $1 \mathrm{~cm}^{2} \times 1 \mathrm{~ns}$. This document studies systematically the gas volume current as a function of the electric field applied across the gas volume and of temperature both with and without the Large Hadron Collider (LHC) beam induced background and up to an instantaneous luminosity $\mathrm{L}_{\text {inst }}=2 \times 10^{34} \mathrm{~cm}^{-2} \mathrm{~s}^{-1}$ (twice larger than the design LHC luminosity). These measurements have been used to study the RPC working conditions and to extrapolate the detector response to the High-Luminosity LHC regime with $\mathrm{L}_{\mathrm{inst}}=7.5 \times 10^{34} \mathrm{~cm}^{-2} \mathrm{~s}^{-1}$.
\end{abstract}

KEYwords: LHC, ATLAS, Trigger detectors, Resistive-plate chambers

${ }^{1}$ Corresponding author. 


\section{Contents}

1 Introduction 1

2 Results with LHC beam induced background 2

3 Results without LHC beam induced background 4

4 Conclusion 6

\section{Introduction}

Muonic signatures play a key role in the physics program of the ATLAS experiment [1] at the Large Hadron Collider (LHC). Resistive Plate Chambers (RPCs) provide the barrel region of the ATLAS detector with an independent muon trigger as well as azimuthal coordinates of muon tracks. The RPC system scheme of the ATLAS experiment is shown in Figure 1.

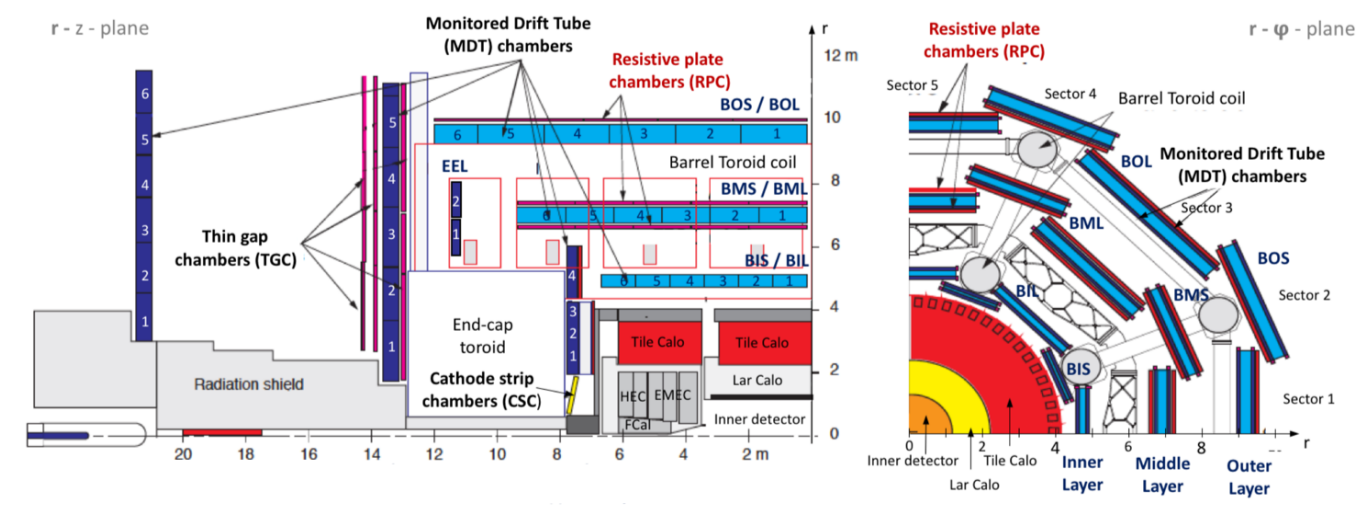

Figure 1. Scheme showing the RPC system of the ATLAS experiment. The detector is segmented in 16 sectors in the $\phi$ direction and 6 stations in the $\eta$ direction on either side of the detector, referred to as A and $\mathrm{C}$ sides. Drawings from [2] modified with respect to those originally found in [1]. Copyright 2020 CERN for the benefit of the ATLAS Collaboration. CC-BY-4.0 license.

Each RPC detector is made of two bakelite plates with a $2 \mathrm{~mm}$ gap, between which the operation gas is located. The gas mixture consists of tetrafluoroethane (94.7\%), iso-butane (5.0\%) and sulphur hexafluoride $(0.3 \%)$. The detector is operated in avalanche mode at $9.6 \mathrm{kV}$ with an automatic correction for temperature and pressure variations with respect to the reference values of $24^{\circ} \mathrm{C}$ and 970 mbar. The signal from the gas volume, induced on pick-up copper strips, is read out via capacitive coupling, and detected by the front-end electronics. Read-out strips have a typical width of $\approx 30 \mathrm{~mm}$ and are grouped in $\eta$ and $\phi$ read-out panels with strips orthogonal to 
each other. Custom read-out electronics amplify, discriminate and convert the detector signals to emitter-coupled logic standard. The RPC is equipped with a Detector Control System [3] to monitor and safely operate around 380k readout channels. More details about the RPC can be found in Reference [4].

Efficient gas volume performance in various LHC running conditions is crucial for detector operation. The gas volume current is used in these studies to check the stability of the RPC as a function of the instantaneous luminosity ( $\left.\mathrm{L}_{\text {inst }}\right)$. Measurements of the gas volume current as a function of the electric field applied across the gas and of the temperature form the basis for the extrapolations of the RPC response to the High-Luminosity LHC (HL-LHC) regime. For studies with the LHC beam induced background, proton-proton data collected at $\sqrt{s}=13 \mathrm{TeV}$ during the second data-taking period of the LHC in 2018 are used.

\section{Results with LHC beam induced background}

The current is obtained individually for each gas volume, by measuring the voltage across a 100 $\mathrm{k} \Omega$ resister in serial connection with the gas volume and ground. For uniformity all currents are normalized to the gas volume area. Currents in gas volumes measured at the working RPC voltage but without proton-proton collisions are subtracted to show the net effect of the luminosity. The RPCs disconnected from the high voltage (HV) or those exhibiting low efficiencies are excluded from the analysis. These amount to about $10 \%$ of the chambers.
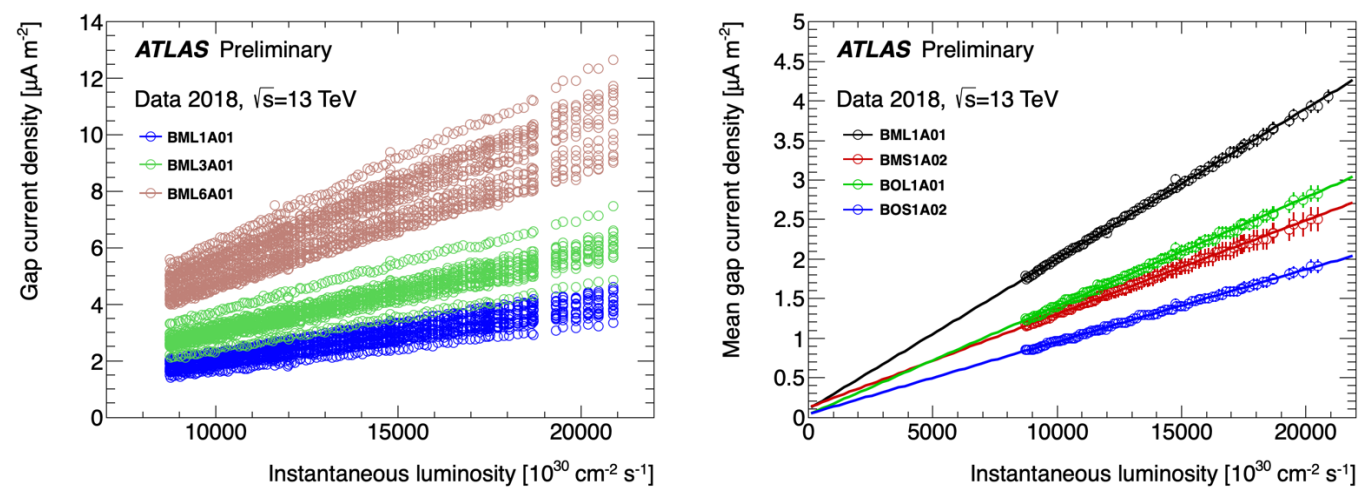

Figure 2. The left plot shows the gas volume current densities as a function of $\mathrm{L}_{\text {inst }}$ in $\eta$ stations 1,3 and 6 (16 gas volumes per station) in the Barrel Middle Large (BML) RPC chambers in side A and $\phi$ sector 1 . The right plot shows averaged current densities over gas volumes in the $\eta$ station 1 in the Barrel Middle/Outer Large/Small (BML/BMS, BOL/BOS) RPC chambers in side A and $\phi$ sectors 1 or 2 as a function of $\mathrm{L}_{\text {inst }}$ [5]. Copyright 2020 CERN for the benefit of the ATLAS Collaboration. CC-BY-4.0 license.

The expected linear increase of the gas volume current densities is observed during stable RPC operation with $\mathrm{L}_{\text {inst }}$ up to $2.1 \times 10^{34} \mathrm{~cm}^{-2} \mathrm{~s}^{-1}$. Figure 2 (left) shows the gas volume current densities as a function of $\mathrm{L}_{\text {inst }}$ for individual RPCs. Their increase with $\eta$ is due to thinner shielding materials in the forward region of the RPC. Additionally, charged particles at a larger $\eta$ have a longer track length in the gas volume, producing more gas ionization. Figure 2 (right) shows averaged current 
densities over gas volumes for various RPC chambers in the $\eta$ station 1 as a function of $\mathrm{L}_{\text {inst }}$, shown separately for the middle (BMx) and outer (BOx) layers, and large (L) and small (S) chamber types.

This plot shows that the gas volume current density decreases with increasing distance from the proton-proton collision point. This effect is explained by the particle flux reduction at larger radii. Discrepancies in current densities between small and large chambers in the same layer are explained by their location with respect to the barrel coils, which systematically shadow the small sectors. Additionally small chambers are located at slightly larger radii from the interaction point than large chambers.

Results shown in Figure 2 confirm the expected performance in the highest luminosity conditions during the second period of LHC operation and allow to extrapolate the gas volume current density to the HL-LHC stage, where $\mathrm{L}_{\text {inst }}$ is expected to reach $\sim 7.5 \times 10^{34} \mathrm{~cm}^{-2} \mathrm{~s}^{-1}$.

RPC current densities have been measured at different operating voltages. The range, from $9.0 \mathrm{kV}$ up to $9.6 \mathrm{kV}$, is chosen to address future possible RPC working conditions. All the current measurements are scaled to the same $\mathrm{L}_{\mathrm{inst}}=1.8 \times 10^{34} \mathrm{~cm}^{-2} \mathrm{~s}^{-1}$, with the largest correction of $3 \%$. Voltages are also corrected for temperature and pressure effects. Each current density measurement is performed twice to estimate a systematic uncertainty. This uncertainty is defined as half of the difference between the two current density measurements at the same voltage.

Figure 3 (left) shows the gas volume current densities as a function of the voltage for 3 individual BML RPC gas volumes in different $\eta$ stations and the same $\phi$ sector. It is observed that the gas volume current during the beam collisions increases with the high voltage approximately linearly and uniformly across the whole RPC detector in the voltage range from 9.0 to $9.6 \mathrm{kV}$. Therefore an extrapolation of the gas volume current can be performed individually per gas volume or with one global scale factor (SF). A comparison between these two procedures is shown in Figure 3 (right). A global SF of 2.1 is defined as the mean value of the ratio, per gas volume, of the current densities at $9.6 \mathrm{kV}$ and $9.2 \mathrm{kV}$. As it can be seen from the plot individual scaling provides a more accurate extrapolation for current densities in this voltage range.

Figure 4 (left) shows distributions of the gas volume current densities for the whole RPC system during data-taking in 2018. Very few gas volumes have a current density higher than $12 \mu \mathrm{A} / \mathrm{m}^{2}$ at $9.6 \mathrm{kV}$ and $\mathrm{L}_{\mathrm{inst}}=1.8 \times 10^{34} \mathrm{~cm}^{-2} \mathrm{~s}^{-1}$. To build the extrapolations to the HL-LHC regime, gas volume current densities measured at $9.2 \mathrm{kV}$ and $9.6 \mathrm{kV}$ are scaled by a factor 4.2 , coming from the ratio between $\mathrm{L}_{\text {inst }}=7.5 \times 10^{34} \mathrm{~cm}^{-2} \mathrm{~s}^{-1}$ and $1.8 \times 10^{34} \mathrm{~cm}^{-2} \mathrm{~s}^{-1}$. Figure 4 (right) shows the resulting distributions of extrapolated current densities for the whole RPC system. Some gas volume current densities exceed $30 \mu \mathrm{A} / \mathrm{m}^{2}$, as reported in the Technical Design Report for the Phase-II Upgrade of the ATLAS Muon Spectrometer [6] as a safe limit for RPC. The overcurrent in the affected gas volumes can be addressed by lowering the voltage from $9.6 \mathrm{kV}$ to $9.2 \mathrm{kV}$. This change will lead to a loss in efficiency that can partly be recovered by adjusting the thresholds of the discriminator [8].

Figure 5 shows the expected gas volume current densities for the HL-LHC program as a function of $\eta$ and $\phi$ for gas volumes belonging to the middle layer (averaged over two sub-layers) at $\mathrm{V}=9.2 \mathrm{kV}$ (left) and $9.6 \mathrm{kV}$ (right). Expectations are based on individual linear fit approximations. These two-dimensional $\eta-\phi$ maps show in details hot gas volumes at $9.6 \mathrm{kV}$.

Figure 5 also shows that the expected current density slightly decreases for the gas volumes located at the bottom of the ATLAS detector, which is compatible with the temperature gradient in 

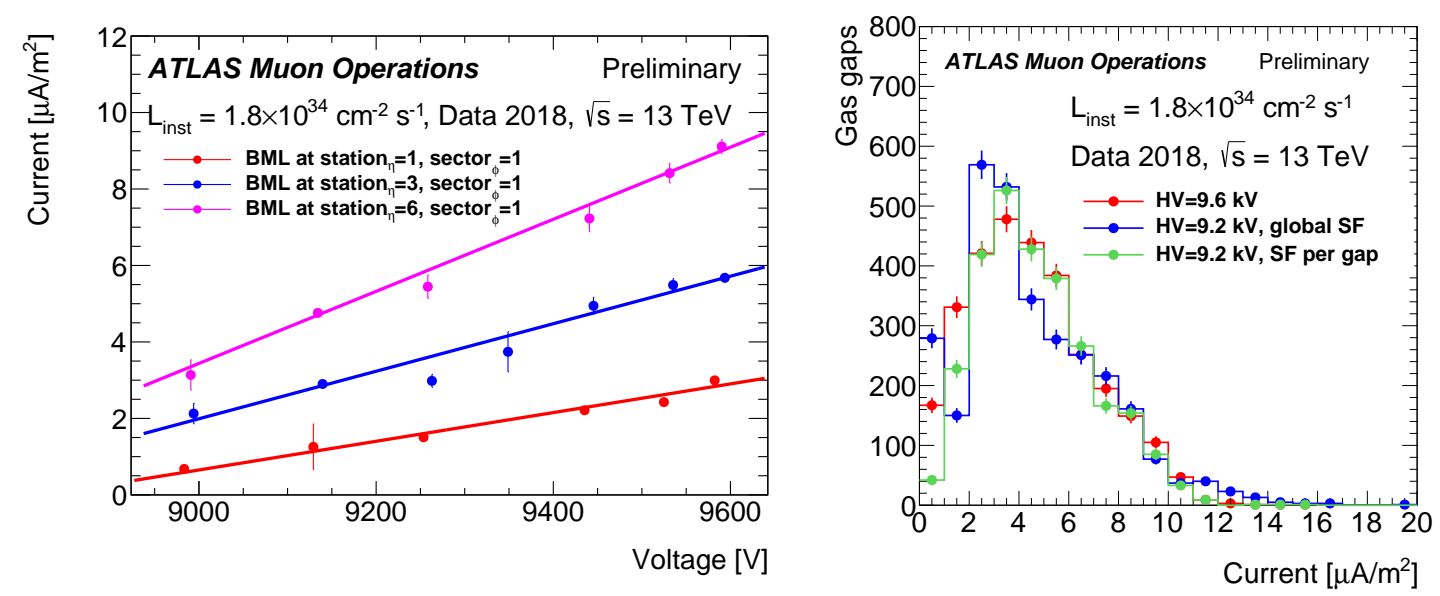

Figure 3. The left plot shows gas volume current densities as a function of the voltage for 3 individual large chambers in different $\eta$ stations, in the same $\phi$ sector. The right plot shows the gas volume current density distribution for the entire RPC obtained directly at $\mathrm{V}=9.6 \mathrm{kV}$ and compared to measurements at $\mathrm{V}=9.2 \mathrm{kV}$ scaled to $9.6 \mathrm{kV}$ [7]. Copyright 2020 CERN for the benefit of the ATLAS Collaboration. CC-BY-4.0 license.
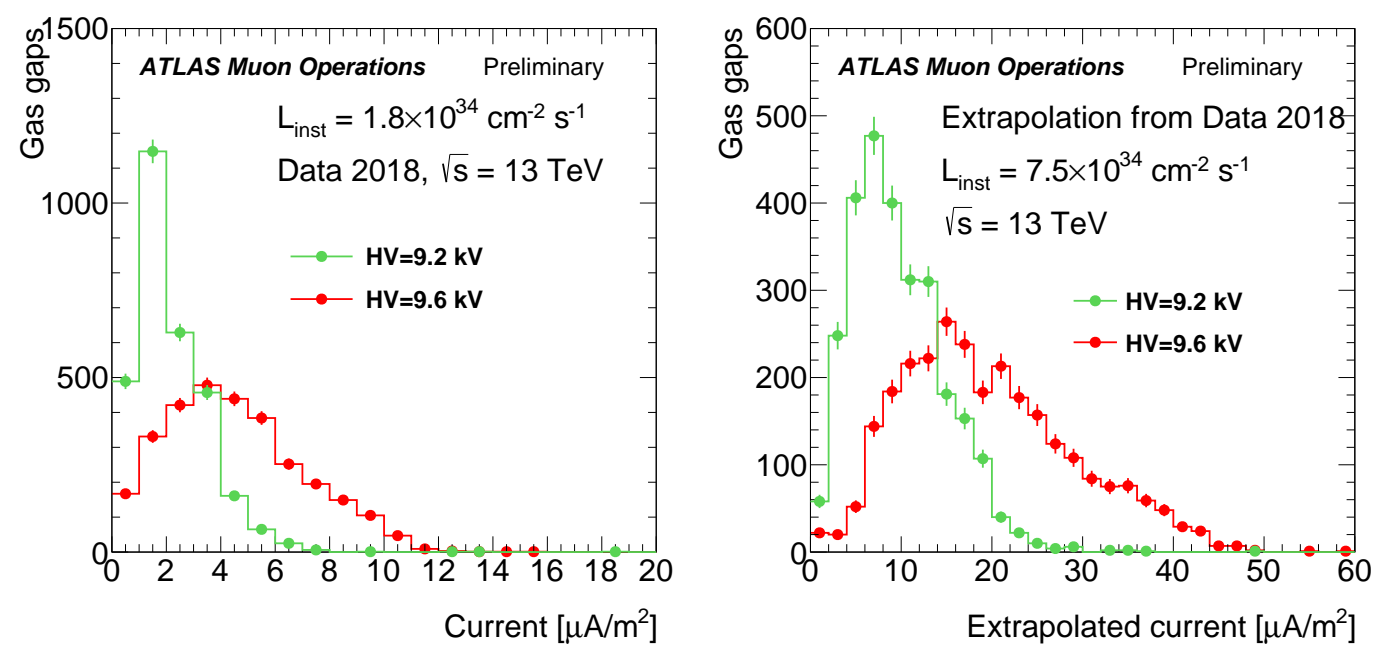

Figure 4. Distribution of gas volume current densities for the full ATLAS RPC system during data-taking in 2018 (left) and extrapolated distribution for $\mathrm{L}_{\mathrm{inst}}=7.5 \times 10^{34} \mathrm{~cm}^{-2} \mathrm{~s}^{-1}$ (right) [7]. Copyright $2020 \mathrm{CERN}$ for the benefit of the ATLAS Collaboration. CC-BY-4.0 license.

the cavern. Studies of this effect show consistent increase of the gas volume current density across the whole RPC of about $6 \mu \mathrm{A} / \mathrm{m}^{2}$ per $10^{\circ} \mathrm{C}$. Figure 6 shows current densities for the RPC BML gas volumes averaged over all $\phi$ sectors in $\eta$ stations $1,4,6$ as a function of the temperature.

\section{Results without LHC beam induced background}

Gas volume current density studies without proton-proton collisions provide a unique and efficient instrument to monitor the state of the gas volumes. It is found that through the entire detector the 

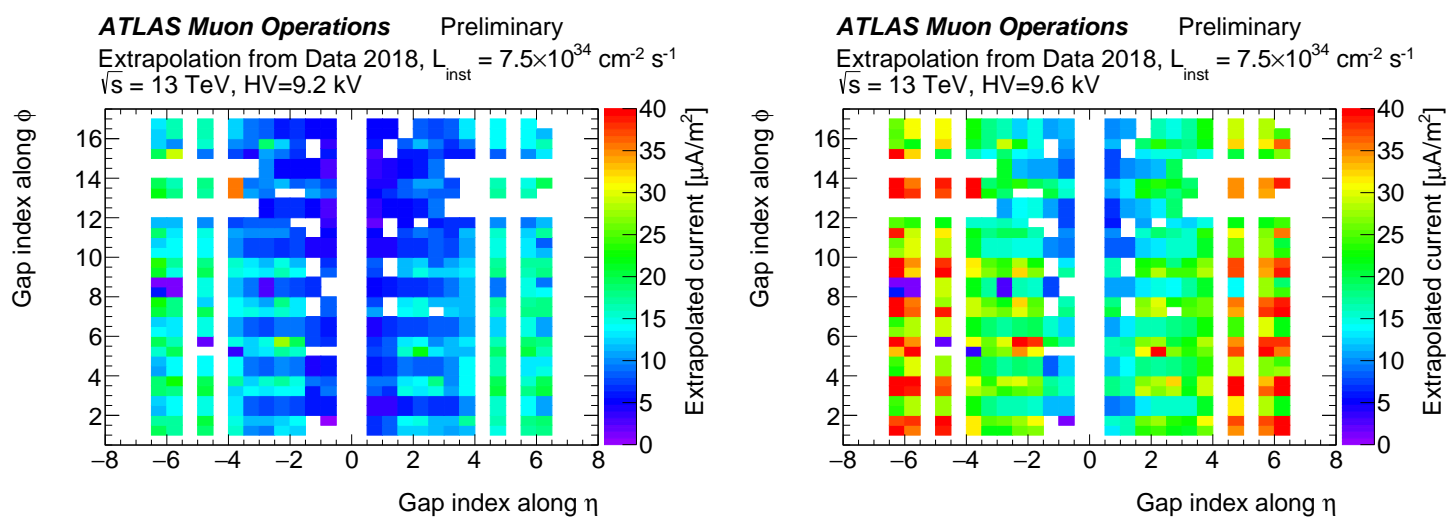

Figure 5. Extrapolated current densities for the HL-LHC program as a function of $\eta$ and $\phi$ for gas volumes belonging to the middle layer (averaged over two sub-layers) at $\mathrm{V}=9.2 \mathrm{kV}$ (left) and $9.6 \mathrm{kV}$ (right) [7]. Copyright 2020 CERN for the benefit of the ATLAS Collaboration. CC-BY-4.0 license.

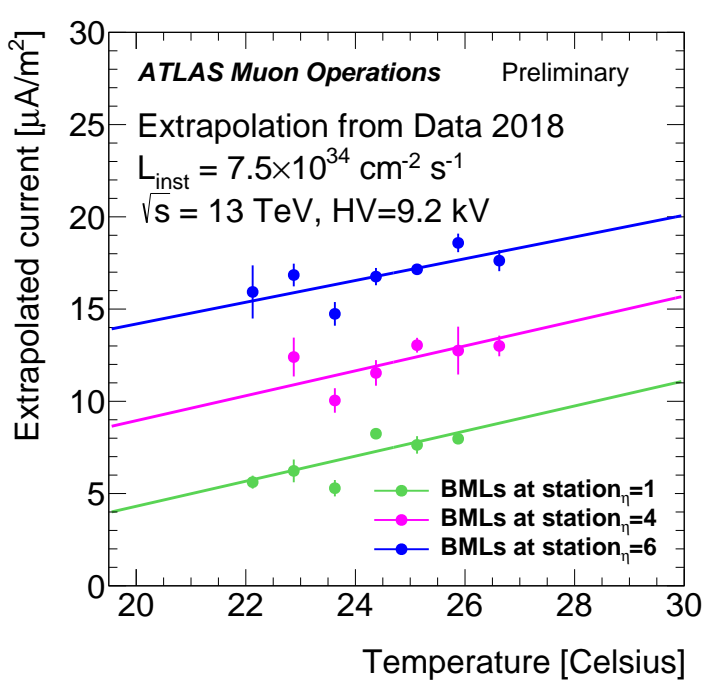

Figure 6. Gas volume current density for RPC middle large gas volumes (BML) averaged over all $\phi$ sectors in $\eta$ stations 1, 4, 6 as a function of the temperature [7]. Copyright 2020 CERN for the benefit of the ATLAS Collaboration. CC-BY-4.0 license.

mean of the gas volume current density distribution without beam induced background increases by $15 \%$ from $\mathrm{V}=9.2 \mathrm{kV}$ to $\mathrm{V}=9.6 \mathrm{kV}$, where a $5 \%$ increase comes from the ohmic contribution, while the other $10 \%$ are from the avalanche growth. These results are shown in Figure 7 (left) and confirm the overall good quality of gas volumes in the RPC detector.

It is found that the dependency of the gas volume current on the voltage at these conditions can be linearly described up to $\mathrm{V}=6 \mathrm{kV}$, then the current density starts to increase exponentially with the voltage. Figure 7 (right) shows the gas volume current density as a function of the voltage for 2 individual RPC middle large gas volumes without beam induced background. The discrepancies 
between these two curves indicate a difference in operation mode of these gas volumes. In general, discrepancies in linear slopes $(\mathrm{V}<6 \mathrm{kV})$ are connected to a different level of cleanliness or deposition on the frame and spacer surfaces within the gas gap volume.
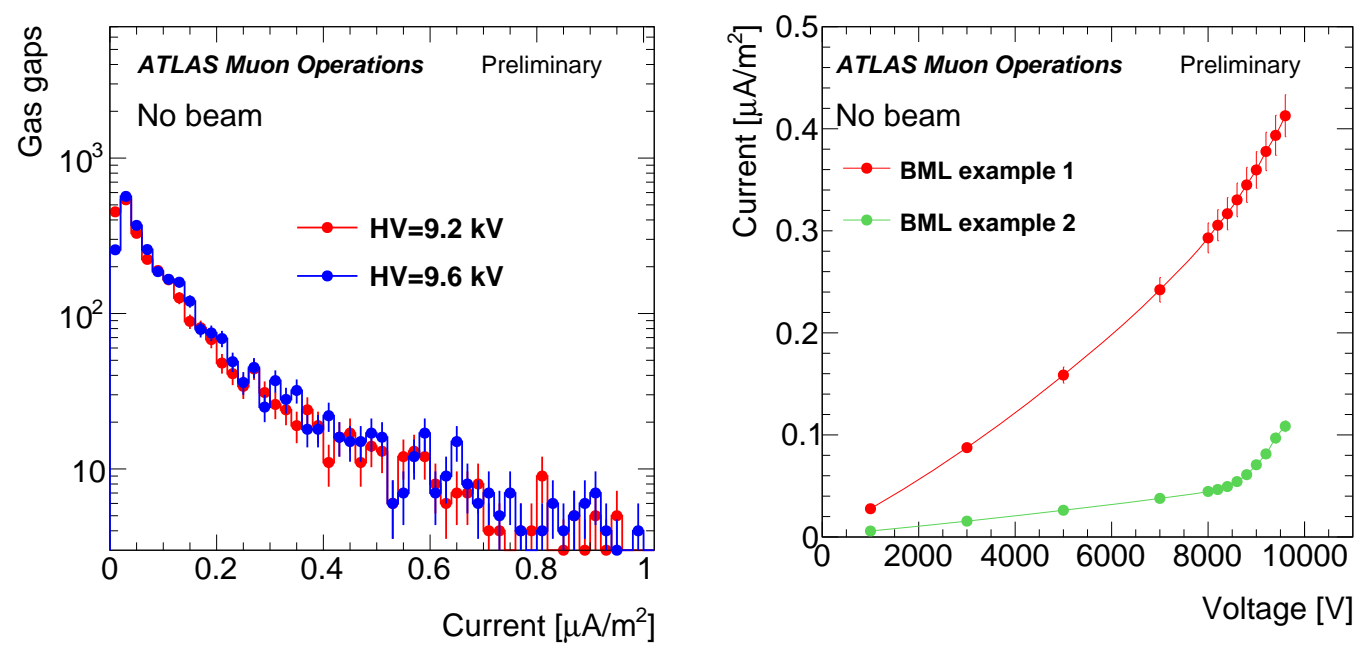

Figure 7. The left plot shows the gas volume current density distribution for the full ATLAS RPC system at two different voltages. The right plot shows the gas volume current density as a function of the voltage for 2 individual RPC middle large gas volumes. Both plots are made without LHC beam induced background [7]. Copyright 2020 CERN for the benefit of the ATLAS Collaboration. CC-BY-4.0 license.

\section{Conclusion}

RPC gas volume current densities are studied as a function of the instantaneous luminosity, voltage and temperature with and without LHC beam induced background. Studies at various luminosities and fixed voltage confirm the expected linear increase of the gas volume current density up to the highest instantaneous luminosity during the second data-taking period in 2018 at the LHC for various RPCs. Results show that the dependency of the gas volume current density on the voltage in the beam condition can be linearly described at V=9-9.6 kV. Studies of gas volume current density extrapolations to HL-LHC conditions show that lowering the voltage to $9.2 \mathrm{kV}$ will help address the overcurrent in affected gas volumes at HL-LHC, however with some loss of efficiency. A consistent increase of the gas volume current density with temperature across the whole RPC is observed $(\approx$ $6 \mu \mathrm{A} / \mathrm{m}^{2}$ per $10^{\circ} \mathrm{C}$ ). Studies of gas volume currents as a function of the voltage without LHC beam induced background are an efficient instrument to monitor the state of gas volumes.

\section{References}

[1] ATLAS Collaboration, The ATLAS Experiment at the CERN Large Hadron Collider, JINST 3 (2008) S08003.

[2] ATLAS Collaboration, Commissioning of the ATLAS Muon Spectrometer with Cosmic Rays, Eur. Phys. J. C 70 (2010) 875. 
[3] A. Polini, Design and Performance of the Detector Control System of the ATLAS Resistive-Plate-Chamber Muon Spectrometer, Nucl. Instrum. Meth. A 661 (2012) S15.

[4] ATLAS Collaboration, The Level-1 Trigger Muon Barrel System of the ATLAS experiment at CERN, JINST 4 (2009) P04010.

[5] ATLAS Collaboration, RPC performance plots based on 2018 dataset, https://atlas.web.cern.ch/Atlas/GROUPS/PHYSICS/PLOTS/MUON-2018-09.

[6] ATLAS Collaboration, Technical Design Report for the Phase-II Upgrade of the ATLAS Muon Spectrometer, CERN-LHCC-2017-017, ATLAS-TDR-026, https://cds.cern.ch/record/2285580.

[7] ATLAS Collaboration, Studies of gas gaps current density in the ATLAS RPC detector during 2018 data taking at Large Hadron Collider, https://atlas.web.cern.ch/Atlas/GROUPS/MUON/PLOTS/MUON-2020-001.

[8] L. Heng, Contribution to the XV Workshop on Resistive Plate Chambers and Related Detectors, RPC 2020. 